\begin{tabular}{|c|l|}
\hline Title & Three dimensional observation of Brownian particles under steady shear flow by stereo microscopy \\
\hline Author(s) & Takikawa, Y oshinori; Nunokawa, Takahiro; Sasaki, Y uji; I wata, Makoto; Orihara, Hiroshi \\
\hline Citation & $\begin{array}{l}\text { Physical Review E, 100(2), 022102 } \\
\text { https://doi.org/10.1103PhysRevE.100.022102 }\end{array}$ \\
\hline Issue Date & 2019-08-02 \\
\hline Doc URL & http://hdl.handle.net/2115/75390 \\
\hline Rights & @2019A A merican Physical Society \\
\hline Type & article \\
\hline File Information & PhysRevE.100.022102.pdf \\
\hline
\end{tabular}

Instructions for use 


\title{
Three-dimensional observation of Brownian particles under steady shear flow by stereo microscopy
}

\author{
Yoshinori Takikawa, ${ }^{1}$ Takahiro Nunokawa, ${ }^{2}$ Yuji Sasaki, ${ }^{2}$ Makoto Iwata, ${ }^{1}$ and Hiroshi Orihara $\oplus^{2, *}$ \\ ${ }^{1}$ Department of Physical Science and Engineering, Nagoya Institute of Technology, Nagoya 466-8555, Japan \\ ${ }^{2}$ Division of Applied Physics, Hokkaido University, Sapporo 060-8628, Japan
}

(Received 4 March 2019; revised manuscript received 19 June 2019; published 2 August 2019)

\begin{abstract}
Three-dimensional observation of Brownian particles under shear flow is performed with a stereo microscope to examine the nature of the Brownian motion that occurs in the presence of shear flow. From the threedimensional trajectories of the particles, we clearly demonstrate the occurrence of anomalous diffusion in the flow direction and the coupling of the displacements in the flow and velocity gradient directions. Furthermore, we experimentally obtain the probability distribution function and current density, which also exhibit characteristic features, and compare the obtained results with theoretical results derived using the Fokker-Planck equation.
\end{abstract}

DOI: 10.1103/PhysRevE.100.022102

\section{INTRODUCTION}

The Brownian motion of particles subjected to shear flow has long been investigated both theoretically and experimentally owing to its intriguing properties of nonequilibrium systems. In the equilibrium state in the absence of macroscopic flow, the mean square displacement (MSD) characterizing the particle diffusion can be expressed as $2 D t$ with the diffusion constant $D$ in all directions. In the case of simple shear flow, the MSD of a particle in the flow direction ( $x$ axis) is modified as

$$
\left\langle[x(t)-x(0)-\dot{\gamma} z(0) t]^{2}\right\rangle=2 D t+\frac{2}{3} D \dot{\gamma}^{2} t^{3},
$$

where the $z$ axis is taken to be along the velocity gradient and $\dot{\gamma}$ is the shear rate. Note that the displacement due to the shear flow along the stream line, $\dot{\gamma} z(0) t$, is subtracted. This is a remarkable feature that originates from coupling between the diffusion due to Brownian motion and the flow. This coupling was first proposed by Taylor, who verified both experimentally and theoretically that the diffusion constant of a particle in a fluid flowing through a narrow tube becomes larger than that in the equilibrium state [1]. The anomalous diffusion under simple shear flow has been theoretically studied using the Langevin equation [2-4] and the convective diffusion equation (Fokker-Planck equation) $[2,5,6]$. However, experimental verification of the anomalous diffusion proportional to $t^{3}$ in Eq. (1) remains difficult, because it requires the measurement of both the $x$ and $z$ coordinates. In a typical experimental setup, only the $x$ and $y$ coordinates can be measured. This difficulty in measuring both the $x$ and $z$ coordinates was overcome by introducing the variable $\tilde{x}(t)=$ $\{[x(2 t)-x(t)]-[x(t)-x(0)]\} / \sqrt{2}$, which contains only $x$ [7]. It is readily apparent that the displacement due to the shear flow in the time interval $t$ is canceled out in $\tilde{x}(t)$. The MSD of $\tilde{x}(t)$ was proved to be the same as that given in Eq. (1): $\left\langle\tilde{x}(t)^{2}\right\rangle=2 D t+(2 / 3) D \dot{\gamma}^{2} t^{3}$. The occurrence of anomalous

\footnotetext{
*orihara@eng.hokudai.ac.jp
}

diffusion was then successfully demonstrated by calculating $\left\langle\tilde{x}(t)^{2}\right\rangle$ solely from measured values of $x(t)$.

Another remarkable feature of Brownian particles under shear flow is that cross-correlation appears between the displacements in the $x$ and $z$ directions, as demonstrated by both theory [2,8] and simulation [9]. Ziehl et al. reported the occurrence of cross-correlation by experimentally obtaining the cross-correlation functions $\langle x(t) z(0)\rangle$ and $\langle z(t) x(0)\rangle$ of a laser-trapped particle under shear flow [10]. Furthermore, this study revealed the breaking of the time-reversal symmetry, i.e., $\langle x(t) z(0)\rangle \neq\langle z(t) x(0)\rangle$. This symmetry breaking is caused by a nonconservative force due to the rotational component of the shear flow. Such a nonconservative force has also been demonstrated to play an important role in orientational fluctuations in sheared nematic liquid crystals $[11,12]$. In the case of a free particle under shear flow, however, there is no experiment to reveal the appearance of the cross-correlation owing to the experimental difficulty mentioned above. In this case, there is no alternative way such as using $\tilde{x}(t)$, and therefore we need to directly observe both the $x$ and $z$ coordinates.

In the probability space for the particle displacement, on the other hand, there are other testable predictions. Theoretically, the probability density was derived from the convective diffusion equation, and anisotropic diffusion was shown to appear under shear flow $[2,5,6]$. However, there has been no experimental verification of these predictions. In addition, we can expect to observe a rotational probability current [13] caused by a nonconservative force. Stereo microscopy allows us to observe these phenomena characteristic to sheared Brownian motion.

In general, to measure three-dimensional (3D) microscale flows, two techniques have mainly been used so far: stereo micro particle tracking velocimetry (PTV) and particle image velocimetry (PIV) [14-17]. In our case, however, the latter is not available, as the velocity of each particle cannot be obtained in the PIV: It gives only the velocity field by calculating the correlation function of successive images. The PTV is the only method that can measure the trajectory of each particle, which has information about not only the velocity 


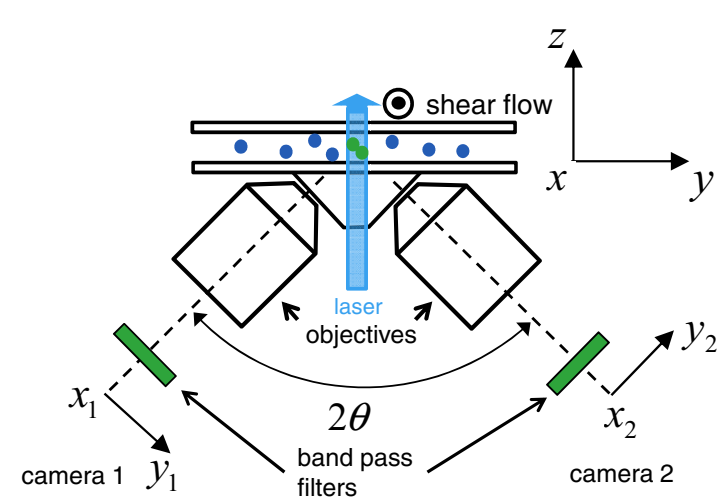

FIG. 1. Schematic illustration of the stereo microscope and shear flow path. Fluorescent particles illuminated with a 488-nm laser were monitored from two distinct optical axes using two identical video microscopes to construct three-dimensional images. The $x, y$, and $z$ axes correspond to the flow, vorticity, and velocity gradient directions, respectively. In stereo microscopy, 3D particle position $(x, y, z)$ is determined by 2D particle-image positions $\left(x_{1}, y_{1}\right)$ and $\left(x_{2}, y_{2}\right)$ taken with cameras 1 and 2 , respectively.

field of the fluid but also the Brownian motion. Therefore, we used microscopic PTV. In the 3D reconstruction from two images taken by two cameras having different optical axes, a precise calibration is necessary. In calibration a calibration grid is usually used. However, this becomes difficult inside a submillimeter-sized cell used in microscopic stereoscopy. So far some calibration procedures for microstereo PTV and PIV have been developed. Here, we present a simple procedure without using any calibration grid.

\section{EXPERIMENT}

To accomplish the 3D monitoring of Brownian particles under shear flow, we employed a stereo particletracking method. Two identical video microscopes with long working distance objectives (magnification: $20 \times$, NA: 0.28 , focal length: $10 \mathrm{~mm}$, working distance: $30.5 \mathrm{~mm}$ ) (MXG-10C, Hirox) equipped with high-sensitivity digital cameras (ORCA-Flash 4.0 V2, Hamamatsu Photonics) were used to simultaneously record distinct off-axial views of the same region of interest, as depicted in Fig. 1. The crossing angle $2 \theta$ between the optical axes was $54^{\circ}$, which permitted measurement of the particle positions in the direction of sample thickness. Fluorescent particles with a diameter of $1 \mu \mathrm{m}$ (FluoSpheres, Invitrogen) dispersed in distilled water were illuminated with a 488-nm laser beam with a diameter of $0.7 \mathrm{~mm}$ (85-BCD-020-053, Melles Griot) and the resulting fluorescent images were captured with a frame rate of $50 \mathrm{fps}$ after passing through a long-pass filter (BLP01-488R-25, Semrock). In total, 8000 frames were acquired for each run and ten runs were performed under the same conditions. Shear flow was applied using a cone-plate rheometer (MCR 302 WESP, Anton Paar) with a glass stage for sample observation. Measurements were done at shear rates of 1 and $2 \mathrm{~s}^{-1}$. We show only results measured at $2 \mathrm{~s}^{-1}$ as the shear effect is more conspicuous at higher shear rates and the results measured at $1 \mathrm{~s}^{-1}$ were consistent with those at $2 \mathrm{~s}^{-1}$. The diameter and cone angle of the cone plate were $25 \mathrm{~mm}$ and $1.134^{\circ}$, respectively, and the samples were observed $2.7 \mathrm{~mm}$ from the center of the upper rotating disk, where the gap was $52 \mu \mathrm{m}$. All measurements were performed at $25^{\circ} \mathrm{C}$. The $3 \mathrm{D}$ trajectories of the particles were obtained from the two movies taken with the two cameras, as follows.

In stereo microscopy, each camera measures the particle displacement perpendicular to its viewing direction. In a coordinate system shown in Fig. 1, the real particle displacement $(\Delta x, \Delta y, \Delta z)$ is given by the particle-image displacements $\left(\Delta x_{1}, \Delta y_{1}\right)$ and $\left(\Delta x_{2}, \Delta y_{2}\right)$ :

$$
\begin{aligned}
& \Delta x=\left(\Delta x_{1}+\Delta x_{2}\right) / 2, \\
& \Delta y=\left(\Delta y_{1}-\Delta y_{2}\right) /(2 \sin \theta), \\
& \Delta z=\left(-\Delta y_{1}+\Delta y_{2}\right) /(2 \cos \theta),
\end{aligned}
$$

for a paraxial approximation, which is valid when the particle displacements are much smaller than the distance to the objectives, and for an ideal experimental setup. In reality, however, a mapping function, which relates $(\Delta x, \Delta y, \Delta z)$ to $\left(\Delta x_{1}, \Delta y_{1}, \Delta x_{2}, \Delta y_{2}\right)$, is experimentally obtained by using a calibration grid because precise calibration is necessary to match particles in the two simultaneous images and obtain the $3 \mathrm{D}$ particle position. In the usual procedure, 3D trajectories are reconstructed by tracking $3 \mathrm{D}$ particles thus obtained. Here, we adopt an alternative procedure without using any calibration grid. We match two-dimensional (2D) trajectories instead of particles. For small displacements, $\left(\Delta x_{1}, \Delta y_{1}, \Delta x_{2}, \Delta y_{2}\right)$ is a linear function of $(\Delta x, \Delta y, \Delta z)$, and therefore a linear relation should hold among $\Delta x_{1}, \Delta y_{1}, \Delta x_{2}$, and $\Delta y_{2}$ by eliminating $\Delta x, \Delta y$, and $\Delta z$; i.e., $\Delta x_{1}=a \Delta x_{2}+$ $b \Delta y_{1}+c \Delta y_{2}$. It is noted that $a=1$ and $b=c=0$ for a perfect match of experimental identification of $\Delta x$ in both cameras. If two $2 \mathrm{D}$ trajectories $\left[\Delta x_{1}(t), \Delta y_{1}(t)\right]$ and $\left[\Delta x_{2}(t), \Delta y_{2}(t)\right]$ originate from an identical 3D trajectory $[\Delta x(t), \Delta y(t), \Delta z(t)], \Delta x_{1}(t)=a \Delta x_{2}(t)+b \Delta y_{1}(t)+$ $c \Delta y_{2}(t)$ holds at all times. We selected best matches in all the pairs of trajectories based on a least squares fit, where we minimized the sum of squared residuals defined as $S=\sum_{i=1}^{N}\left[\Delta x_{1}\left(t_{i}\right)-a \Delta x_{2}\left(t_{i}\right)-b \Delta y_{1}\left(t_{i}\right)-c \Delta y_{2}\left(t_{i}\right)\right]^{2} / N$. Although this method is applicable only to moving particles, it is quite useful for our purpose. The 3D displacements were calculated by using Eq. (2) without any calibration. No calibration means that the 3D displacements would contain some errors. For example, an error of $1^{\circ}$ in $\theta$ causes errors of about $3 \%$ and $1 \%$ in $\Delta y$ and $\Delta z$, respectively. Taking into account the measurement error, we confine ourselves to mainly investigating the qualitative properties of sheared Brownian motion in this paper.

\section{EXPERIMENTAL RESULTS AND DISCUSSION}

\section{A. Generalized MSD, cross-correlation, and asymmetric interaction}

In Fig. 2, we show the 3D trajectories observed under shear flow for a typical single run, where only 40 trajectories were randomly selected, and the $x$ and $z$ directions correspond to the flow and velocity gradient directions, respectively, and short trajectories have been removed. The observed region was approximately $360 \times 80 \times 20 \mu \mathrm{m}^{3}$ in the $x, y$, and $z$ directions, 


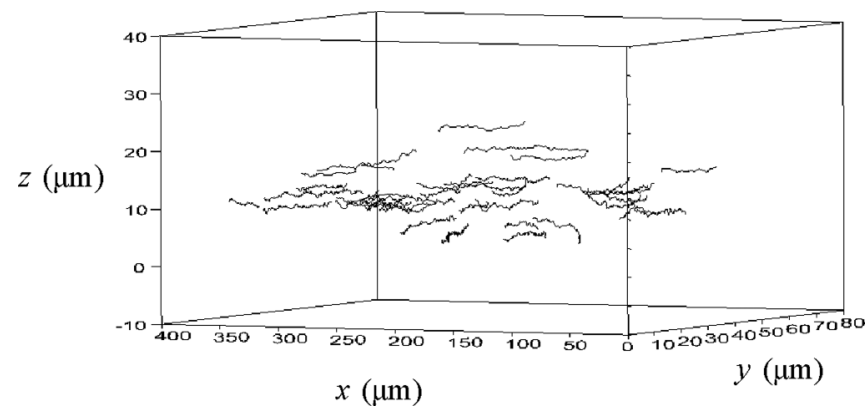

FIG. 2. Typical 3D trajectories observed using the stereo microscope. The observed region is approximately $360 \times 80 \times 20 \mu \mathrm{m}^{3}$ in the $x, y$, and $z$ directions, where $z$ ranges from 15 to $35 \mu \mathrm{m}$ and the bottom glass surface is at $z=0$. A simple shear flow of $1.9 \mathrm{~s}^{-1}$ is applied in the $x$ direction.

and especially $z$ approximately ranged from 0 to $20 \mu \mathrm{m}$, where the glass surface was at $z=0$. In principle, the sizes of the region in the $y$ and $z$ directions were determined by the depth of field of the objectives, the crossing angle $2 \theta$, and the availability of the matching procedure. Although there were particles moving near the glass surface, the amount of particles between $z=0$ and $5 \mu \mathrm{m}$ was only $8 \%$ so that the wall effect could be neglected in our experiment. It can be clearly seen that the fluctuating particles were carried away in the flow direction. In the previous section we referred only to the displacements, but the particle positions are shown in Fig. 2. They were obtained by using $\left(x_{1}, y_{1}, x_{2}, y_{2}\right)$ instead of $\left(\Delta x_{1}, \Delta y_{1}, \Delta x_{2}, \Delta y_{2}\right)$ in Eq. (2), and the $z=0$ surface corresponding to the glass surface was determined by the velocity field, which becomes zero at the surface and was obtained from the trajectories as follows. Along each trajectory, we obtained the velocities at an interval of $0.2 \mathrm{~s}$. By assuming a flow velocity field of $\mathbf{v}(\mathbf{r})=\left\{\dot{\gamma} \mathbf{g} \cdot\left[\mathbf{r}-\mathbf{r}_{0}\right]\right\} \mathbf{f}$, where $\mathbf{f}$ and $\mathbf{g}$ are the unit vectors in the flow and velocity gradient directions, respectively, and $\dot{\gamma}$ is the shear rate regarded as an adjustable parameter, we fitted the equation to the set of calculated velocities at various positions. The total number of trajectories used for the least-squares fit was 1702 over ten runs. The obtained shear rate $\dot{\gamma}$ was $1.9 \mathrm{~s}^{-1}$, which is slightly less than the value of $2 \mathrm{~s}^{-1}$ set using the rheometer. The vectors $\mathbf{f}$ and $\mathbf{g}$ slightly deviated from the $x$ and $z$ directions, respectively, and therefore the coordinate axes were redefined such that the $x$ and $z$ axes were parallel to $\mathbf{f}$ and $\mathbf{g}$.

Here, we define the generalized MSD:

$$
\begin{aligned}
M_{\alpha \beta}(t) \equiv & \left\langle\left\{r_{\alpha}(t)-r_{\alpha}(0)-v_{\alpha}[\vec{r}(0)] t\right\}\right. \\
& \left.\times\left\{r_{\beta}(t)-r_{\beta}(0)-v_{\beta}[\vec{r}(0)] t\right\}\right\rangle
\end{aligned}
$$

It should be noted that $M_{\alpha \beta}(t)$ is a symmetric tensor in general, $M_{\alpha \beta}(t)=M_{\beta \alpha}(t)$, and $v_{\alpha}(\mathbf{r})=\dot{\gamma} z \delta_{\alpha x}$ in the present case. The diagonal elements (usual MSDs) $M_{\alpha \alpha}(t)$ are theoretically obtained as

$$
\begin{gathered}
M_{x x}(t)=\left\langle[x(t)-x(0)-\dot{\gamma} z(0) t]^{2}\right\rangle=2 D t+\frac{2}{3} D t(\dot{\gamma} t)^{2}, \\
M_{y y}(t)=M_{z z}(t)=2 D t,
\end{gathered}
$$

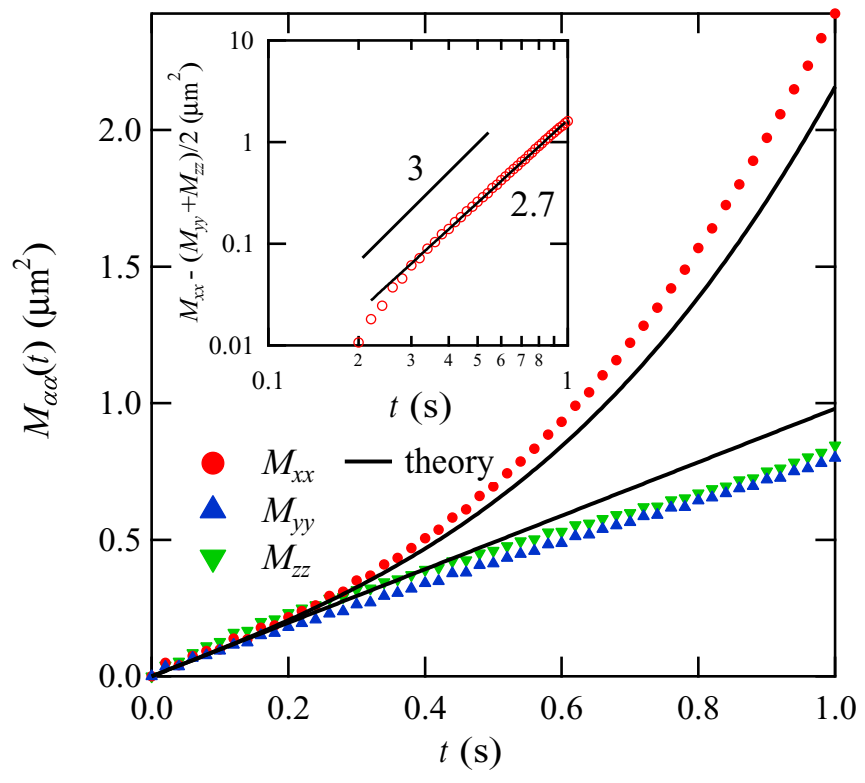

FIG. 3. Diagonal elements $M_{\alpha \alpha}(t)(a=x, y, z)$ for Brownian particles with a diameter of $1 \mu \mathrm{m}$ under shear flow at a shear rate of $1.9 \mathrm{~s}^{-1}$ at $25{ }^{\circ} \mathrm{C}$ in distilled water. The solid lines represent the theoretical results. Anomalous diffusion is observed in the $x$ direction $\left[M_{x x}(t)\right]$, which can be seen more clearly in the log-log plot of $M_{x x}-\left(M_{y y}+M_{z z}\right) / 2$ shown in the inset. The slope is 2.7 , which is slightly smaller than the theoretical value 3 . In contrast, normal diffusion is observed in the $y$ and $z$ directions.

and the off-diagonal elements (cross-correlations) are obtained as [2]

$$
\begin{gathered}
M_{x z}(t)=\langle[x(t)-x(0)-\dot{\gamma} z(0) t][z(t)-z(0)]\rangle=D \dot{\gamma} t^{2}, \\
M_{x y}(t)=M_{y z}(t)=0 .
\end{gathered}
$$

These results can be calculated from the following Langevin equation:

$$
\zeta\left(\frac{d r_{\alpha}}{d t}-\delta_{x \alpha} \dot{\gamma} z\right)=R_{\alpha}(t),
$$

where $\zeta=6 \pi a \eta$ with $a$ the particle radius and $\eta$ the shear viscosity, and the diffusion constant is given by the EinsteinStokes relation $D=k_{B} T / \zeta . R_{\alpha}$ is the Gaussian white noise: $\left\langle R_{\alpha}(t)\right\rangle=0$ and $\left\langle R_{\alpha}(t) R_{\beta}\left(t^{\prime}\right)\right\rangle=2 \zeta k_{B} T \delta_{\alpha \beta} \delta\left(t-t^{\prime}\right)$. As mentioned above, in the flow direction the $t^{3}$ term is added to the MSD [Eq. (4)], and cross-correlation occurs between the $x$ and $z$ directions, which is proportional to $t^{2}$ [Eq. (6)]. This cross-correlation originates from the coupling between $x$ and $z$, although it is asymmetric as shown by Eq. (8): $x$ depends on $z$ but $z$ is independent of $x$, which will be demonstrated experimentally.

The diagonal elements $M_{\alpha \alpha}(t)$ are plotted in Fig. 3, where the solid lines were obtained theoretically using Eqs. (4) and (5). The diffusion constant in these equations was calculated using the Einstein-Stokes relation with $a=0.5 \mu \mathrm{m}$ and $\eta=$ $0.89 \mathrm{mPa} \mathrm{s}$ at $25{ }^{\circ} \mathrm{C}$. Normal diffusion was observed in both the $y$ and $z$ directions, although the experimental MSDs 


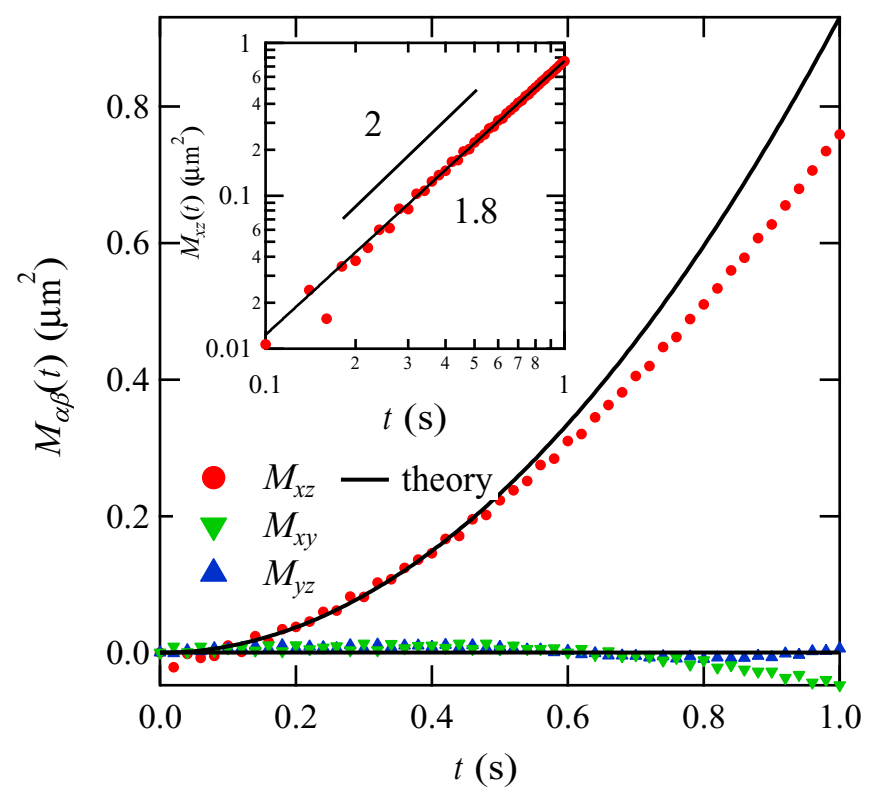

FIG. 4. Off-diagonal elements $M_{\alpha \beta}(t)(\alpha \neq \beta)$ calculated using the same data as in Fig. 3. The solid lines represent the theoretical results. A correlation is observed between $x$ and $z\left[M_{x z}(t)\right]$, which obeys a power law with an exponent of 1.8 slightly smaller than the theoretical value 2 (see the inset).

were slightly lower than the theoretical values. This discrepancy may be mainly attributable to the experimental error, which was caused by using Eq. (2) without any calibration. In the flow direction, in contrast, anomalous diffusion was clearly observed. The inset of Fig. 3 shows a plot of $M_{x x}-$ $\left(M_{y y}+M_{z z}\right) / 2$, obeying a power law with an exponent of 2.7 , which is slightly smaller than the theoretical value 3 . The discrepancy may be due to the above-mentioned experimental error and/or another error contained in the linear flow velocity field used in the calculation. These results are consistent with those described in our previous report [7], in which the MSD of $\tilde{x}(t)$ was considered.

The off-diagonal elements $M_{\alpha \beta}(t)(\alpha \neq \beta)$ are plotted in Fig. 4. The curves for $M_{x y}$ and $M_{y z}$ were almost zero. In contrast, the curve for $M_{x z}$ was not zero and obeys a power law with an exponent of 1.8 (see the log-log plot shown in the inset), which is slightly smaller than the theoretical value 2 . The reason for the discrepancy may be the same as that in $M_{x x}$. These results clearly demonstrate the correlation between $x$ and $z$.

In the case of a trapped particle, the breakdown of the timereversal symmetry was demonstrated through the relation $\langle x(t) z(0)\rangle \neq\langle z(t) x(0)\rangle$ [10]. In the case of our unbounded particles, however, $\langle x(t) z(0)\rangle$ and $\langle z(t) x(0)\rangle$ are ill-defined. Instead, we consider

$$
\begin{gathered}
\tilde{M}_{x z}(t) \equiv\langle[x(t)-x(0)-\dot{\gamma} z(0) t][z(2 t)-z(t)]\rangle, \\
\tilde{M}_{z x}(t) \equiv\langle[z(t)-z(0)][x(2 t)-x(t)-\dot{\gamma} z(0) t]\rangle .
\end{gathered}
$$

These are calculated as $\tilde{M}_{x z}(t)=0$ and $\tilde{M}_{z x}(t)=2 D \dot{\gamma} t^{2}$ using Eq. (8). The former is obvious because $x(\tau)(0 \leqslant \tau \leqslant t)$ never influences $z(\tau)(t \leqslant \tau \leqslant 2 t)$, whereas $z(\tau)(0 \leqslant \tau \leqslant t)$

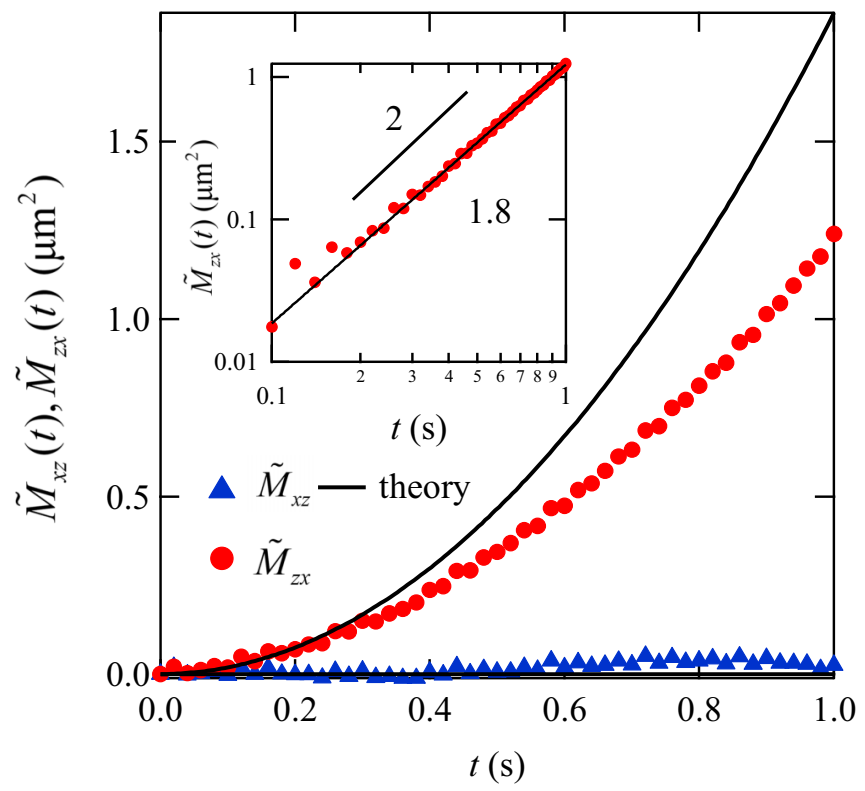

FIG. 5. Plots of $\tilde{M}_{x z}(t)$ and $\tilde{M}_{z x}(t)$. The solid lines represent the theoretical results. The results for $\tilde{M}_{x z}(t)=0$ and $\tilde{M}_{z x}(t) \neq 0$ indicate the existence of an asymmetric interaction between $x$ and $z$. From the inset the slope is 1.8 , which is slightly smaller than the theoretical value 2 .

influences $x(\tau)(t \leqslant \tau \leqslant 2 t)$, which originates from the asymmetric interaction between $x$ and $z$ as previously discussed. This is demonstrated in Fig. 5. The curve for $\tilde{M}_{x z}(t)$ is almost zero and that for $\tilde{M}_{z x}(t)$ obeys a power law with an exponent of 1.8 , which is slightly smaller than the theoretical value 2 . The reason for the discrepancy may be the same as described above. Although $\tilde{M}_{x z}(t)$ and $\tilde{M}_{z x}(t)$ correspond to $\langle x(t) z(0)\rangle$ and $\langle z(t) x(0)\rangle$, respectively, there is a crucial difference with respect to time reversal. The time reversal of $\tilde{M}_{x z}$ corresponds to $\langle[x(2 t)-x(t)-\dot{\gamma} z(2 t) t][z(t)-z(0)]\rangle=0$ and that of $\tilde{M}_{z x}(t)$ to $\langle[z(2 t)-z(t)][x(t)-x(0)-\dot{\gamma} z(2 t) t]\rangle=-2 D \dot{\gamma} t^{2}$, which are obtained by the replacements $x(t) \rightarrow x(-t), z(t) \rightarrow$ $z(-t)$, and $\dot{\gamma} \rightarrow-\dot{\gamma}$ and by using time translation symmetry. Therefore, $\tilde{M}_{x z}(t)$ and $\tilde{M}_{z x}(t)$ are not mutually transformed by time reversal, whereas $\langle x(t) z(0)\rangle$ and $\langle z(t) x(0)\rangle$ are mutually transformed.

\section{B. Probability distribution function and current density}

Next, we consider the Brownian motion under shear flow in probability space. The probability density $P(\mathbf{r}, t)$ obeys the Fokker-Planck equation [2]:

$$
\frac{\partial P(\mathbf{r}, t)}{\partial t}=-\nabla \cdot \mathbf{J}(\mathbf{r}, t),
$$

where the probability current is given in our case as

$$
\mathbf{J}(\mathbf{r}, t)=-D \nabla P(\mathbf{r}, t)+\mathbf{v}(\mathbf{r}, t) P(\mathbf{r}, t),
$$

with

$$
\mathbf{v}(\mathbf{r}, t)=(\dot{\gamma} z, 0,0) .
$$

The first and second terms of the right-hand side of Eq. (12) correspond to the diffusive and convective 
contributions, respectively. From Eq. (8), $\zeta \mathbf{v}$ can be regarded as an external force, which can be decomposed into elongational and rotational components, $\zeta(\dot{\gamma} z / 2,0, \dot{\gamma} x / 2)$ and $\zeta(\dot{\gamma} z / 2,0,-\dot{\gamma} x / 2)$, respectively. The former is a conservative force that can be expressed in terms of a potential energy, whereas the latter is a nonconservative force. Nonconservative forces play important roles in nonequilibrium systems [13].

In order to experimentally obtain the probability density at $t=n \Delta t$, where $\Delta t$ is the sampling interval (20 ms in our experiment) and $n$ is an integer, we made a data set consisting of points $[x[(n+m) \Delta t]-x(m \Delta t)-\dot{\gamma} z(m \Delta t) n \Delta t$, $y[(n+m) \Delta t]-y(m \Delta t), z[(n+m) \Delta t]-z(m \Delta t)]$ in the displacement space for possible integers $m$ in each 3D trajectory. The density of particles is proportional to the probability density. In Fig. 6, we plot all the points projected on the planes perpendicular to the $x, y$, and $z$ axes at three different times, together with the corresponding projected probability densities defined as

$$
\bar{P}_{\alpha}\left(r_{\alpha+1}, r_{\alpha+2}, t\right)=\int_{-\infty}^{+\infty} P(\mathbf{r}, t) d r_{\alpha}(\alpha=1,2,3)
$$

with the following analytical solution $[2,6]$ :

$$
\begin{aligned}
P(\mathbf{r}, t)= & \frac{3^{1 / 2}}{4(\pi D t)^{3 / 2}\left(\dot{\gamma}^{2} t^{2}+12\right)^{1 / 2}} \\
& \times \exp \left[-\left\{\frac{3(x-\dot{\gamma} t z / 2)^{2}}{D t\left(\dot{\gamma}^{2} t^{2}+12\right)}+\frac{y^{2}}{4 D t}+\frac{z^{2}}{4 D t}\right\}\right],
\end{aligned}
$$

where the subscripts $\alpha+1$ and $\alpha+2$ indicate the values modulo 3. In the shear plane ( $x-z$ plane) [Fig. 6(a)], the distribution is initially almost isotropic and then anisotropically diffused outward owing to the irrotational contribution. The experimental results are in good agreement with the theoretical ones. In the plane perpendicular to the flow direction $(y-z$ plane) [Fig. 6(b)], the distribution is completely isotropic at all time points, because the diffusion constants in the $y$ and $z$ directions are identical. In contrast, in the $x-y$ plane [Fig. 6(c)], the distribution becomes elongated in the flow direction owing to the anomalous diffusion.

Finally, we discuss the probability current density $\mathbf{J}(\mathbf{r}, t)$, which was obtained by counting the number of particles passing through small areas perpendicular to the $x, y$, and $z$ axes. Figure 7 shows the projected current density on the shear plane $(x-z$ plane), which is defined as

$$
\left(\int_{-\infty}^{+\infty} J_{x}(\mathbf{r}, t) d y, \int_{-\infty}^{+\infty} J_{z}(\mathbf{r}, t) d y\right),
$$

together with the theoretical results calculated from Eqs. (12), (13), and (15). Initially, the current is radially outward owing to the diffusive contribution, and as the convective contribution becomes larger a rotational current originating from the nonconservative force appears. The rotational current appears solely in the shear plane, and only radially outward currents were observed in the other two planes.
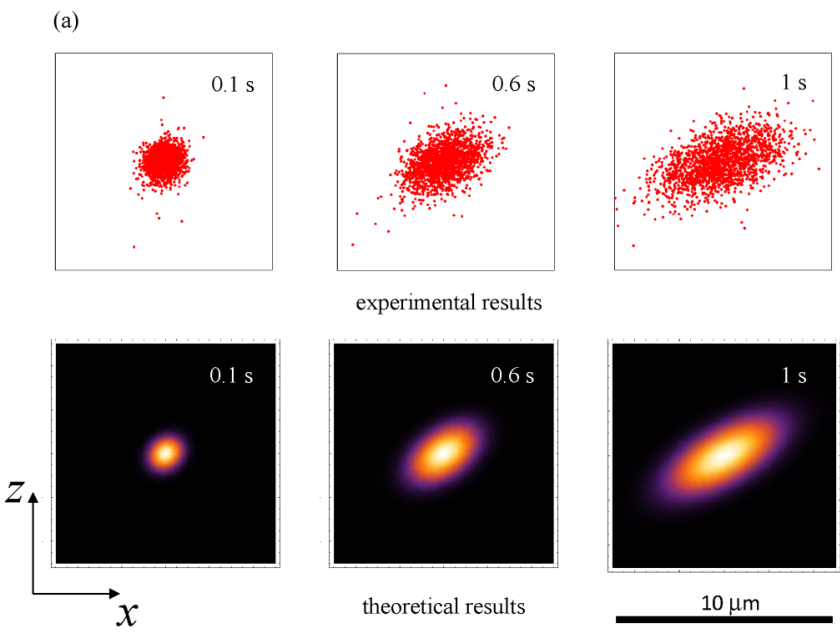

experimental results
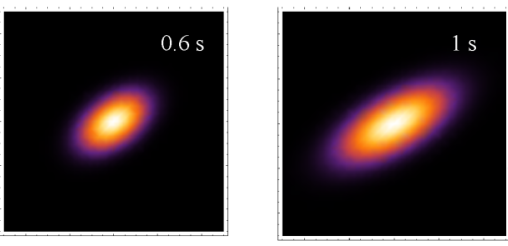

theoretical results

$10 \mu \mathrm{m}$

(b)
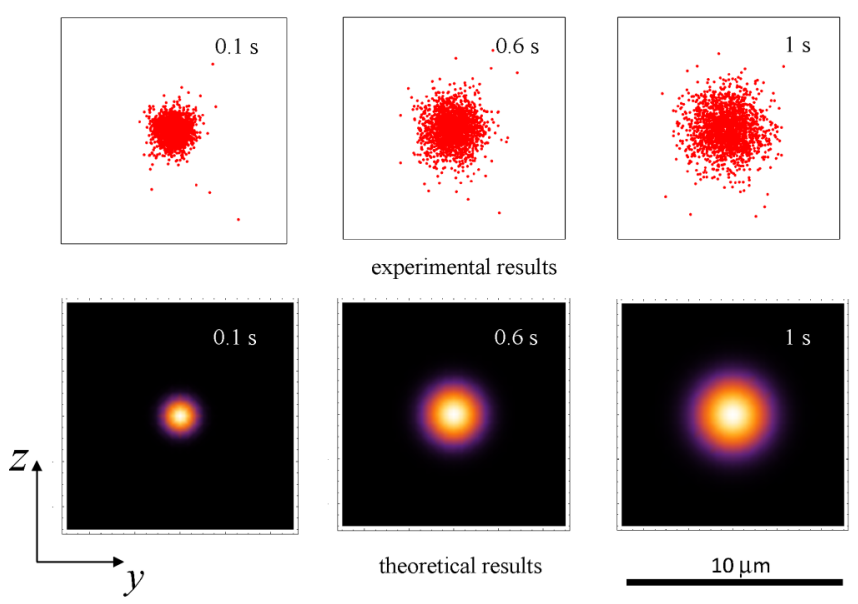

experimental results
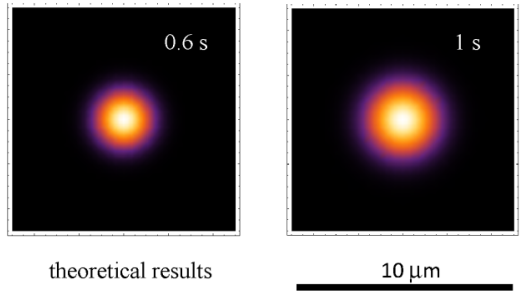

(c)
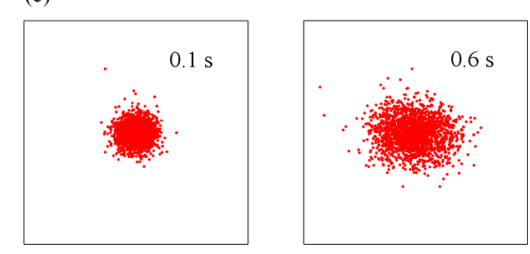

experimental results
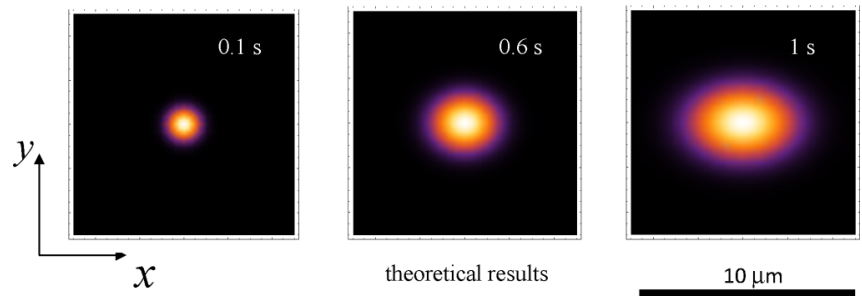

FIG. 6. Experimental (top panels) and theoretical (bottom panels) particle probability densities projected on the planes perpendicular to the (a) $y$, (b) $x$, and (c) $z$ axes. From the trajectories in Fig. 2, displacements were obtained at an interval of $t$, which correspond to points in the 3D displacement space. The projections of the points on the above three planes yield the top panels. The bottom panels were obtained by numerically calculating Eqs. (14) and (15). 


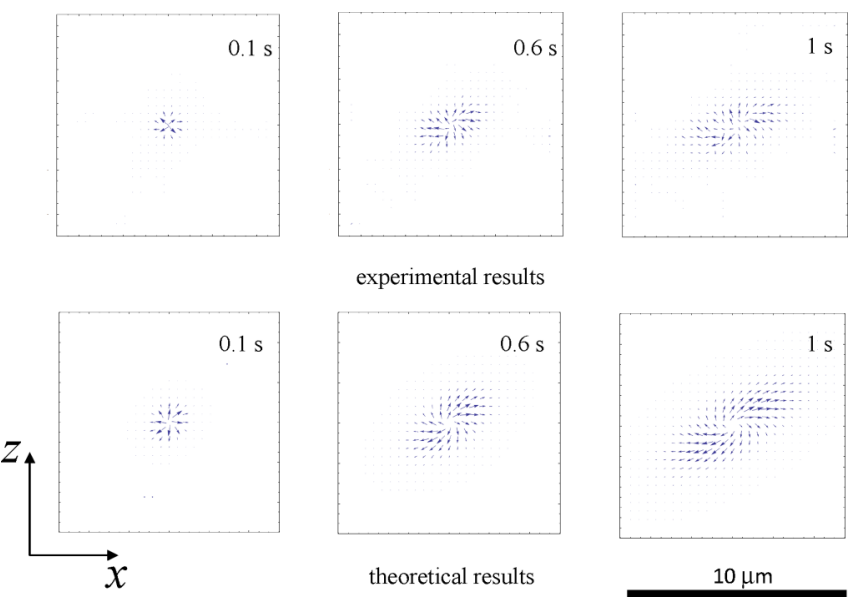

FIG. 7. Experimental (top panels) and theoretical (bottom panels) probability current densities projected on the shear plane $(x-z$ plane). The probability current density was experimentally obtained by counting the number of particles passing through small areas perpendicular to the $x, y$, and $z$ axes. The theoretical results were obtained by numerically calculating Eq. (16). A rotational current originating from the macroscopic rotational fluid flow or nonconservative force is observed.

\section{CONCLUSIONS}

A stereo particle-tracking method was successfully applied to the 3D observation of Brownian particles under steady shear flow. From the three-dimensional trajectories, the macroscopic flow velocity field was determined to calculate generalized MSDs $M_{\alpha \beta}(t)$. The $M_{x x}(t)$ and $M_{x z}(t)$ curves demonstrated, respectively, the anomalous diffusion in the flow direction and the correlation between the displacements in the flow and velocity gradient directions. Furthermore, the asymmetric interaction between $x$ and $z$ was confirmed by demonstrating that $\tilde{M}_{x z}(t) \neq \tilde{M}_{z x}(t)$.

Further analyses were performed in the probability space. We determined the probability distribution function and the current density from the obtained trajectories and found the results to be in good agreement with those calculated using the Fokker-Planck equation. In particular, a characteristic rotational current was observed in the current density projected on the shear plane, which originated from the nonconservative force or macroscopic rotational flow.

At the end of this paper, we would like to mention that our 3D particle-tracking method is applicable also to nonNewtonian fluids, which are expected to show intriguing anisotropic diffusion, though we applied it to a typical Newtonian fluid, water, here. For example, we have recently observed the Brownian motion of particles dispersed in xanthan gum solutions with a 2D particle-tracking method and found that the diffusion in the vorticity direction (the $y$ direction in the present setup) increases as well as in the flow direction (the $x$ direction) under shear flow [18]. Application of our 3D particle-tracking method will reveal the diffusion in the velocity gradient direction (the $z$ direction) and deepen our understanding of anisotropic diffusion in such non-Newtonian fluids.

\section{ACKNOWLEDGMENT}

This work was supported by JSPJ KAKENHI Grant No. JP25103006.
[1] G. Taylor, Proc. R. Soc. London, Ser. A 219, 186 (1953).

[2] R. T. Foister and T. G. M. van de Ven, J. Fluid Mech. 96, 105 (1980).

[3] C. van den Broeck, J. M. Sancho, and M. S. Miguel, Phys. A (Amsterdam, Neth.) 116, 448 (1982).

[4] P. Schram and S. A. Trigger, Phys. B (Amsterdam, Neth.) 228, 91 (1996).

[5] D. E. Elrick, Aust. J. Phys. 15, 283 (1962).

[6] T. G. M. van de Ven, J. Colloid Interface Sci. 62, 352 (1977).

[7] H. Orihara and Y. Takikawa, Phys. Rev. E 84, 061120 (2011).

[8] L. Holzer, J. Bammert, R. Rzehak, and W. Zimmermann, Phys. Rev. E 81, 041124 (2010).

[9] T. Iwashita and R. Yamamoto, Phys. Rev. E 79, 031401 (2009).

[10] A. Ziehl, J. Bammert, L. Holzer, C. Wagner, and W. Zimmermann, Phys. Rev. Lett. 103, 230602 (2009).
[11] H. Orihara, F. Yang, Y. Takigami, Y. Takikawa, and Y. H. Na, Phys. Rev. E 86, 041701 (2012).

[12] J. F. Fatriansyah and H. Orihara, Phys. Rev. E 88, 012510 (2013).

[13] K. Tomita and H. Tomita, Prog. Theor. Phys. 51, 1731 (1974).

[14] J. G. Santiago, S. T. Wereley, C. D. Meinhart, D. J. Beebe, and R. J. Adrian, Exp. Fluids 25, 316 (1998).

[15] R. Lindken, J. Westerweel, and B. Wieneke, 3D micro-scale velocimetry methods: A comparison between $3 \mathrm{D}-\mu \mathrm{PTV}$, stereoscopic $\mu \mathrm{PIV}$ and tomographic $\mu \mathrm{PIV}$, in Proceedings of the 13th International Symposium on Applications of Laser Techniques to Fluid Mechanics, Lisbon, Portugal, 2006, Paper 1099.

[16] S. J. Lee and S. Kim, Microfluid. Nanofluid. 6, 577 (2009).

[17] C. Cierpka, M. Rossi, R. Segura, F. Mastrangelo, and C. J. Kähler, Exp. Fluids 52, 605 (2012).

[18] Y. Takikawa, M. Yasuta, S. Fujii, H. Orihara, Y. Tanaka, and K. Nishinari, J. Phys. Soc. Jpn. 87, 054005 (2018). 